\title{
Analysis on influence of adjacent buildings on mutual seismic acceleration response
}

\author{
Lin Yan ${ }^{1}$, Hui Long ${ }^{1}{ }^{*}$, Shu-wen Liu ${ }^{1}$, Wen-zhao Chen ${ }^{1}$ and Cheng Peng ${ }^{1}$ \\ ${ }^{1}$ College of Civil Engineering, University of South China, Hengyang 421000, China
}

\begin{abstract}
During an earthquake, the seismic response of adjacent buildings will be affected by each other, which is known as structure-soil-structure interaction (SSSI). In order to study the SSSI between neighboring buildings, the slab foundation frame structure on a typical type II site in Nanjing is used as the object. ABAQUS software is used to establish a nonlinear static-dynamic coupling two-dimensional finite element analysis model of a single building and multiple buildings with different spacing. The numerical analysis of the model shows that: Under the action of earthquakes, the increase of surrounding structures will increase its own acceleration response; when the building spacing ratio is $1 / 2$ and $1 / 4$, the existence of adjacent buildings will reduce its own acceleration response. When the spacing ratio is greater than or equal to 1 , the impact tends to be unfavorable, and it is the most unfavorable when the spacing ratio is 2 . When the spacing ratio is greater than or equal to 4 , the impact of adjacent buildings on each other's peak acceleration response can be ignored.
\end{abstract}

\section{Introduction}

The seismic safety of various building structures during an earthquake is very important. Existing research often isolates the research object on the engineering site and only studies the seismic response of the isolated building structure. In fact, most of the time the building does not exist alone. There may be a large number of other building structures around the building, especially in cities, and tightly adjacent buildings inevitably have extremely complex interactions during earthquakes, which is known as structure-soil-structure interaction (SSSI). The current research on SSSI is mainly focused on theoretical derivation and numerical simulation. Finite element numerical analysis method is the main method for analyzing SSSI problems in ground motion ${ }^{[1]}$.Such as S.Wang ${ }^{[2]}$ used FEM-BEM to study the influence of parameters which is building spacing, the alignment direction of the two building foundations and the load position on the dynamic response of the structure. Lehmann $\mathrm{L}$ and Antes $\mathrm{H}^{[3]}$ studied the Earthquake response of two multistory buildings on a rigid foundation, but their research is limited to two buildings. In addition, Padro'n LA ${ }^{[4]}$ analyzed the adjacent buildings under earthquakes by modeling soil and superstructure, They observed that the SSSI is strongly affected by the separation distance. However, in their study, only the equivalent linear model was used for the soil and the dynamic nonlinear characteristics of soil and structure were not considered at the same time. In recent years, the SSSI problem conditions under nonlinear analysis have become the mainstream work of research. For example, Huai-feng Wang ${ }^{[5]}$ and others have studied the SSSI of vertically incident S-waves, considering the nonlinear of soil, but their study did not consider static conditions. Pierfrancesco Cacciola ${ }^{[6]}$ first proposed the use of a SSSI mechanism to develop a device that is located in the soil but separated from the structure, capable of absorbing part of the seismic energy, thereby reducing the vibration of nearby structures. Currently, considerable work is still needed to assess the SSSI effects between study structures.

In this paper, ABAQUS is used to target a raft foundation frame structure on a typical type II site in Nanjing. I built many Non-linear static-dynamic coupling two-dimensional finite element analysis models of multiple building soil-structure systems with different spacing. By comparing the results of numerical simulation analysis, the effects of the number of adjacent buildings and spacing on the acceleration response of the structure are studied. The research conclusions have certain reference significance for the seismic performance evaluation of adjacent building superstructures.

\section{Model parameters}

\subsection{Soil layer parameters}

Taking a typical type II horizontal layered site in Nanjing as the site condition, the thickness of the overburden is $30 \mathrm{~m}$ and the equivalent shear wave velocity is $212.34 \mathrm{~m} / \mathrm{s}$. The distribution and mechanical parameters of the soil layer are shown in Table 1.

\footnotetext{
*Corresponding author: 28748388@qq.com
} 
Table 1. mechanical parameters of each soil layer.

\begin{tabular}{cccccc}
\hline $\mathrm{Z}(\mathrm{m})$ & $\gamma\left(\mathrm{KN} / \mathrm{m}^{3}\right)$ & $V_{\mathrm{S}}(\mathrm{m} / \mathrm{s})$ & $\begin{array}{c}\varphi \\
\left({ }^{\circ}\right)\end{array}$ & $\mathrm{u}$ & $\gamma_{0}$ \\
\hline $0-2.5$ & 18.8 & 141 & 13 & 0.49 & 0.00032 \\
$2.5-9$ & 19.2 & 185 & 19 & 0.49 & 0.00035 \\
$9-17$ & 20.1 & 220 & 22 & 0.49 & 0.00038 \\
$17-24.5$ & 20.3 & 260 & 27 & 0.49 & 0.0004 \\
$24.5-30$ & 20.2 & 237 & 29 & 0.49 & 0.0004 \\
\hline
\end{tabular}

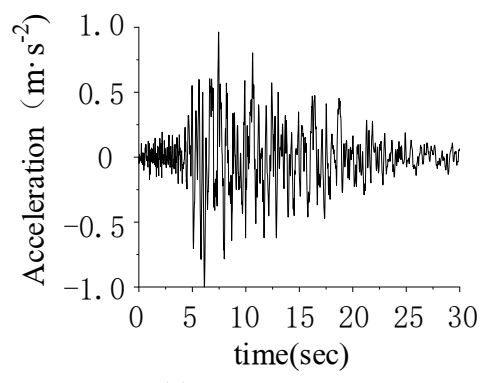

(a).LP wave

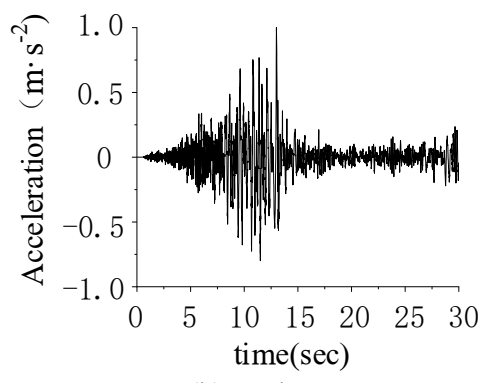

(b).Wolong wave

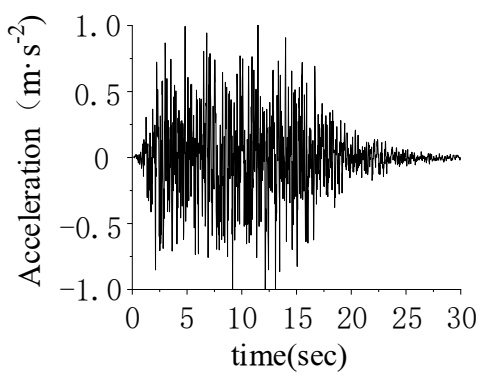

(c).Nanjing wave

Fig.1. Time history of input seismic wave acceleration

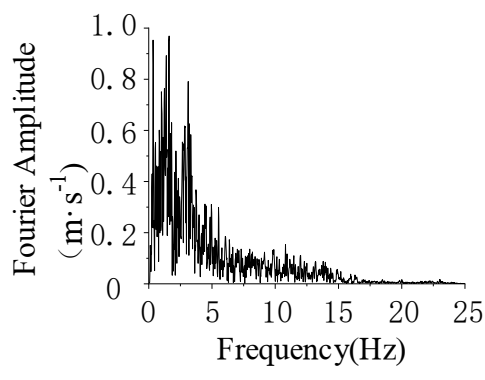

(a).LP wave

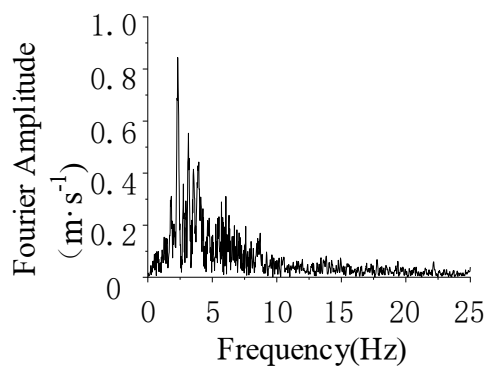

(b).Wolong wave

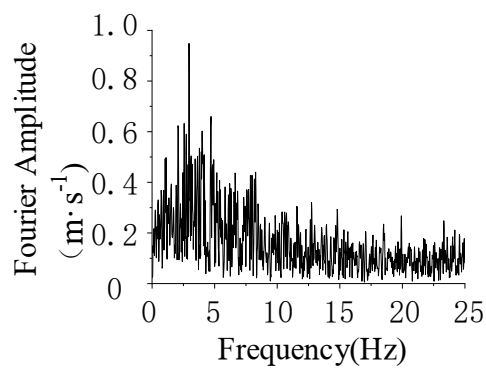

(c).Nanjing wave

Fig.2. Fourier amplitude

near-field seismic wave-Wolong wave (hereinafter referred to as WL) and the Nanjing artificial seismic wave (hereinafter referred to as $\mathrm{NJ}$ ) are selected, with a peak acceleration amplitude of $1.0 \mathrm{~m} / \mathrm{s}^{2}$. The first $30 \mathrm{~s}$ of the duration interception, its acceleration time history curve and Fourier amplitude are shown in Fig.1. and Fig.2.

\section{2D finite element model}

This article takes a raft-frame structure on a typical type II site in Nanjing as an example. The raft foundation is $15 \mathrm{~m}$ wide and $0.6 \mathrm{~m}$ thick, then the buildings is a reinforced concrete structure with 12 floors plus a basement which height is $3.6 \mathrm{~m}$, and The height of the floor is $3 \mathrm{~m}$, of which the thickness is $0.6 \mathrm{~m}$, in addition, the span in the vertical plane direction is $6 \mathrm{~m}$. First, a twodimensional finite element model of building $\mathrm{B}$ and site is established, and then increased Building A and Building $\mathrm{C}$ (exactly the same structure as Building B), and changing the spacing to form two and three Building models. This article mainly considers the influence of the buildings on both sides on the acceleration response of the intermediate building, so this article mainly studies the acceleration change law of Building $B$. Let definition the spacing ratio (the ratio of the building distance to the building foundation width) is $0.25,0.5,1,2,3,4,6$, respectively, as shown in Fig.3.The calculated width of the site is $495 \mathrm{~m}$. In the subsequent analysis, the span is enlarged in proportion for comparison and verification. The raft foundation is enlarged to $22 \mathrm{~m}$, and the corresponding spacing is adjusted according to the spacing ratio, and the site is enlarged to $726 \mathrm{~m}$.

The equal stiffness ratio method is used to turn the three-dimensional problem into a two-dimensional problem. Beam-column members are equivalent to a continuous wall arranged in the vertical plane direction, then the total mass and total lateral stiffness of the wall are guaranteed to be the same as those of the original beam-column system. At the same time, the density of the equivalent rear wall is appropriately increased to take into account the influence of the weight of the infill wall, the weight of the floor and the load on the floor. The concrete uses the plastic damage model of ABAQUS, and the mesh uses CPE4 element. The soil is modeled by the viscoplastic memory-type nested surface constitutive model studied by Professor Zhuang Haiyang, and the soil mesh is simulated by CPE4RH element. The foundation surface is the main surface, and the soil on the side of the foundation is a slave surface. The normal contact method of the contact surfaces is defined as hard contact, when the foundation and soil contact, it is assumed that the normal contact pressure can be transmitted between the 
foundation and soil, and When the pressure is 0 , foundation and soil are separated. The tangential contact method is defined as friction contact, and the friction coefficient is 0.4 . In the static-dynamic coupling analysis, the model is analyzed by a three-step method. First step is use the PREDEFINED FIELDS method to balance crustal stresses. In this step, horizontal displacement is constrained on both sides of the site, and vertical displacement is constrained at the bottom by a fixed boundary. Second step is the transformation of static boundary conditions. Due to the need to remove the horizontal constraints on both sides of the boundary during dynamic analysis, in order to maintain the crustal stress is unchanged, and node concentrated loads which is exactly the same as the boundary node constraint loads is applied to the boundary node to achieve the transition from static analysis to dynamic analysis of the model. Third step is to apply horizontal ground vibration at the bottom of the model for dynamics analysis.

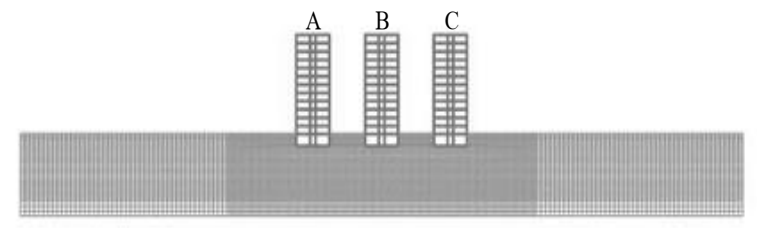

Fig.3. Two-dimensional finite element models for three buildings which spacing ratio is 1

\section{Analysis of calculation results}

Here we define an acceleration effect coefficient $\triangle$,

$$
\triangle=\left(a^{\prime}-a\right) / a
$$

$a^{\prime}$-The peak acceleration of $\mathrm{B}$ building every floor when there are two or three buildings.

$a$-The peak acceleration of a certain floor for a single building (only building B).

The calculation results show that under the input of three seismic waves, the $\triangle$ is ranges from $-13.88 \%$ to $12.08 \%$. The adjacent buildings $\mathrm{A}$ and $\mathrm{C}$ have $\mathrm{a}$ significant impact on the peak acceleration of building $\mathrm{B}$.

\subsection{Building spacing and the effects of seismic waves}

Due to the length of article is limited, the first floor, the fifth floor, and the tenth floor of the building B are selected for this analysis. Fig.4, Fig.5 and Fig.6 show the $\triangle$ in two buildings and three buildings with different spacing ratio, Under the three kinds of earthquake wave. It can be seen that when the spacing ratio is $1 / 4$ and $1 / 2$, the $\triangle$ nearly all are less than 0 , which indicates that the impact on the acceleration peak of building $B$ is advantageous at this time, and the maximum acceleration amplitude can be reduced by $13.88 \%$ when the spacing ratio is $1 / 4$, which may be because the buildings are separated by a certain distance, and The overall effect is formed between buildings, which reduces the acceleration response. When the spacing ratio is greater than or equal to 1 , the $\triangle$ basically are greater than 0 , indicating that the acceleration of building $\mathrm{B}$ is amplified, and the impact tends to be unfavorable. The most adverse effects of each floor basically appear when the spacing ratio is 2 , the maximum acceleration peak is amplified by $12.08 \%$. At this time, the surrounding buildings leads increase in the peak of its own acceleration, which must be given sufficient attention. When the spacing ratio is greater than or equal to 4 , the $\triangle$ almost are within $5 \%$, which indicates that the impact of surrounding buildings on the peak acceleration is very small and can be ignored.

In addition, through these three pictures, it can be seen that under the action of Nanjing artificial seismic wave, the $\triangle$ appear the minimum and maximum values, which is $-13.88 \%$ and $12.08 \%$. It indicate that the Nanjing artificial seismic wave has the largest influence on the acceleration change between adjacent buildings among the three types of seismic waves. Under the action of LP wave ground motion, the $\triangle$ changed is the smallest among the three types of seismic waves, which indicate that the structure is relatively resistant to far-field seismic waves. These may be caused by the relationship between the frequency of the seismic wave and the natural frequency of the structure.

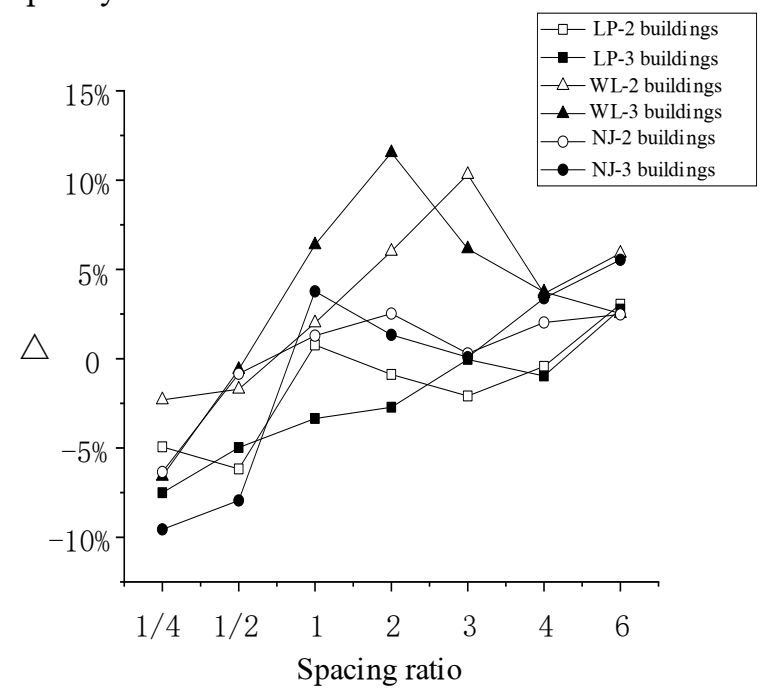

Fig.4. The $\triangle$ of building B's one floor under three seismic waves

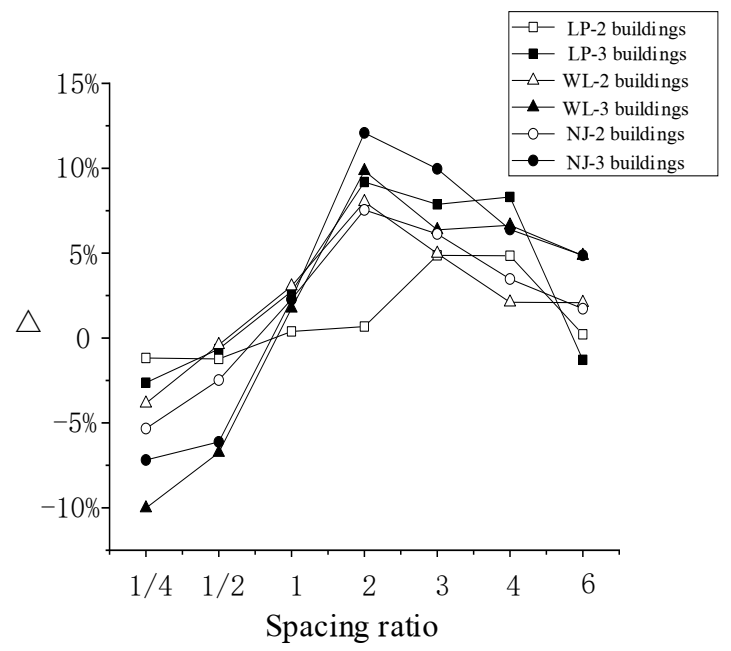


Fig.5. The $\triangle$ of building B's fifth floor under three seismic waves

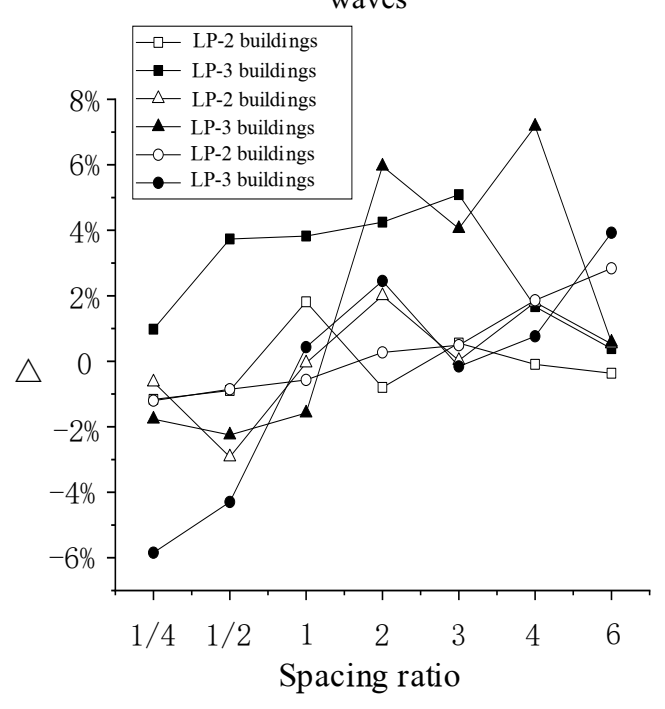

Fig.6. The $\triangle$ of building B's twelve floor under three seismic waves

\subsection{Spacing ratio verification}

Table 2 shows the parts of each floor of building B before and after the three building models are enlarged under the action of LP wave and NJ wave ground motion. Among them, the spacing ratio is given before the enlarged model is 4 , and the spacing is $60 \mathrm{~m}$. Then the spacing ratio is given after the enlarged model is 3 and 4 , at this time, the spacing is $66 \mathrm{~m}$ and $88 \mathrm{~m}$ respectively. It can be seen that after changing the width of the building, when the spacing ratio is 3 , the $\Delta$ is still relatively large at this time, the maximum impact is $-9.54 \%$, which cannot be ignored. When the spacing ratio is 4 , almost the $\triangle$ are within $5 \%$, which further verifies that when the spacing ratio is 4 , the influence of surrounding buildings on its own acceleration peak is very small.

Table 2 The $\triangle$ of each floor of building B before and after the enlarged model in three buildings model (\%)

\begin{tabular}{|c|c|c|c|c|c|c|}
\hline \multirow{2}{*}{ floor } & \multicolumn{3}{|c|}{ LP } & \multicolumn{3}{c|}{ NJ } \\
\cline { 2 - 7 } & before & \multicolumn{2}{|c|}{ after } & before & \multicolumn{2}{c|}{ after } \\
\cline { 2 - 7 } & 4 & 3 & 4 & 4 & 3 & 4 \\
\hline 1 & -0.97 & 7.50 & 3.76 & 3.38 & -9.54 & -4.60 \\
\hline 2 & 6.53 & 8.67 & 5.89 & 4.34 & -1.59 & 3.29 \\
\hline 3 & 4.83 & 8.93 & 1.55 & 4.81 & -0.19 & 2.69 \\
\hline 4 & 4.27 & 7.88 & 0.64 & 6.62 & 8.70 & 3.17 \\
\hline 5 & 3.42 & 6.90 & -0.05 & 6.39 & 8.02 & 0.30 \\
\hline 6 & 6.80 & 5.32 & -0.90 & -1.93 & 6.58 & 4.77 \\
\hline 7 & 4.60 & 3.01 & -1.70 & -1.41 & 3.80 & 6.51 \\
\hline 8 & -0.55 & 2.61 & -2.51 & -5.29 & 1.22 & 6.27 \\
\hline 9 & -1.44 & -3.44 & -1.12 & -2.16 & 1.37 & 2.62 \\
\hline 10 & -1.77 & -2.89 & -3.99 & -1.26 & 0.09 & 4.15 \\
\hline 11 & -1.68 & 4.07 & 0.76 & -0.02 & 3.69 & 5.66 \\
\hline 12 & -0.79 & 6.22 & 0.97 & 0.48 & 6.98 & 5.25 \\
\hline
\end{tabular}

\section{Conclusion}

Through the calculation and analysis of this article, the following conclusions can be drawn:

(1) In these earthquake, the different distance between adjacent buildings will have favorable or unfavorable effect on the peak acceleration response of the intermediate building. When the building spacing ratio is $1 / 4$ and $1 / 2$, the effect will tend to be favorable, and when the spacing ratio is $1 / 4$, it appears the largest influence coefficient for $13.88 \%$. When the building spacing ratio is greater than 1, the impact tends to be unfavorable. what is more, when the spacing ratio is 2 , it is the most unfavorable and the acceleration amplitude is maximized which can reach $12.08 \%$, it will have a negligible impact on the earthquake resistance of the structure. When the spacing ratio is greater than or equal to 4 , the influence of the adjacent buildings on the acceleration of the intermediate building can be ignored.

(2) In this example, the Nanjing artificial earthquake wave has the largest impact on the acceleration amplitude between adjacent buildings among the three types of seismic waves, and the LP wave has the smallest impact, indicating that the different seismic waves will also affect the peak acceleration response of intermediate buildings.

\section{Acknowledgments}

Hunan Provincial Natural Science Fund, 2017JJ3263; National Natural Science Foundation of China(NSFC), 51708273

\section{References}

1. Li-ping Dou, Dan-guan Pan. Seismic response analysis of adjacent structures under the action of SSSI. Building Structure, 2013. (in Chinese)

2. Wang S, Schmid G. Dynamic structure-soil-structure interaction by FEM-BEM. Computational Mechanics, 1992.

3. Lehmann L, Antes H. Dynamic structure-soilstructure interaction applying the symmetric Galerkin boundary method (SGBEM), 2001.

4. Padro'n LA, Azna'rez JJ, Maeso O. Dynamic structure-soil-structure interaction between nearby piled buildings under seismic excitation by BEMFEMmodel,2009.

5. Wang H F, Lou M L, Zhang R L. Influence of presence of adjacent surface structure on seismic response of underground structure, Soil Dynamics and Earthquake Engineering, 2017.

6. Pierfrancesco Cacciola, Maria-Garcia Espinosa, Alessandro Tombari. Vibration control of piledstructures through structure-soil-structure-interaction, Soil Dynamics and Earthquake Engineering,2015.

7. Hai-yang Zhuang, Guo-xing Chen, Ding-hua Zhu. Dynamic viscoplastic memory nested surface constitutive model of soil and its verification, Chinese Journal of Geotechnical Engineering, 2006. (in Chinese) 
8. Hui Long, Guo-xing Chen, Hai-yang Zhuang. Effective stress analysis of seismic response characteristics of subway station structures on liquefiable foundations, Rock and Soil Mechanics, 2013. (in Chinese)

9. Guo-xing Chen, Lei Chen, Li-ping Jing, Hui Long. Comparison of Explicit and Implicit Algorithms for Parallel Calculation of Seismic Analysis of Underground Structures of Metros,2010(in Chinese)

10. GB 50011-2016.Code for seismic design of buildings, China Construction Industry ,2016(in Chinese) 